\title{
Alzheimer's disease in a patient with posterior cortical atrophy
}

\author{
Marcelo L Berthier, Ramon Leiguarda, Sergio E Starkstein, Gustavo Sevlever, \\ Ana L Taratuto
}

\begin{abstract}
Posterior cortical atrophy (PCA) is characterised by slowly progressive dementia with cognitive and perceptual deficits suggestive of bilateral parietooccipital disease. A case is reported of a patient with PCA and neuropathological findings consistent with Alzheimer's disease.
\end{abstract}

Benson et $a^{1}$ recently described posterior cortical atrophy (PCA) in five patients who showed slowly progressing dementia with initial alexia and visual agnosia, followed by Balint's syndrome, Gertsmann's syndrome, constructional apraxia, spatial disorientation, and transcortical sensory aphasia. All patients had bilateral parieto-occipital atrophy. On neuropathological examination one patient showed bilateral parietal subcortical gliosis and another showed bilateral parietal congophilic angiopathy, neuritic plaques (NPs) and neurofibrillary tangles (NFTs). ${ }^{2}$

Our patient with neuropathologically verified Alzheimer's disease (AD) presented with cognitive deficits restricted to functions served by the posterior parietal and occipital lobes.

\section{Case report}

Neurology Service, Hospital Clinic and Provincial, Barcelona, Spain

M Berthier

Institute of Neurological Research

"Dr Raul Carrea"

Buenos Aires,

Argentina

$R$ Leiguarda

G Sevlever

A L Taraturo

Department of

Psychiatry and

Behavioral Sciences,

The Johns Hopkins

University School of

Medicine, Baltimore,

Maryland, USA

S E Starkstein*

University of Buenos Aires, ^ Buenos Aires, Argentina

Correspondence to: Dr Berthier, Servicio de Neurologia, Hospital Clinic Provincial, Villarroel 170 (08036), Barcelona, Espana

Received 29 January 1991. Accepted 26 April 1991
The patient was a 48 year old, right handed woman with 12 years of formal education, who had a three year history of slowly progressive visual loss and writing difficulties. Her father had had dementia and a sister had multiple sclerosis. One year before the examination she gradually became unable to read and write, developed problems in manual skills and calculation, and showed episodes of spatial disorientation. Her main visual complaint was piecemeal perception and transient disappearance of objects. Although she could identify objects by sight, her relatives reported that she behaved like a blind person as she could not navigate her room without colliding with objects. These disturbances led to early retirement. Her relatives also noticed that she developed an anxiety disorder in the absence of prominent language or memory deficits. On neurological examination she was alert and oriented. Though ocular fundus, visual fields, oculocephalic movements, and optokinetic responses were all normal, saccadic eye movements to the left were slow. Visual acuity was difficult to assess due to abnormal fixation, and her corrected visual acuity for both eyes was $6 / 10,8 / 10$, and $4 / 10$ in three successive evaluations. Results of the rest of the neurological examination were unremarkable.

On neuropsychological evaluation her Blessed dementia scale ${ }^{3}$ score was $5 \cdot 5$ points, and the Mini-Mental State Examination ${ }^{4}$ score was 22 points. On the WAIS, ${ }^{5}$ there was a remarkable dissociation between verbal IQ (87 points) and the performance IQ ( 0 points) (the patient could not perform any of the subtests). On the Weschler memory scale, ${ }^{6}$ her memory quotient was below average ( 80 points); she showed severe impairment of visual memory for geometric designs, but her immediate verbal recall and overall verbal memory were only marginally defective. Language was fluent and grammatically correct but contained occasional semantic paraphasic substitutions. Auditory comprehension and sentence repetition were normal. She had echolalia, however, impaired object naming, and defective word list generation. On the Western aphasia battery, ${ }^{7}$ she scored 84.4 points, and the profile was consistent with a mild anomic aphasia. She also showed letter-by-letter reading, impaired

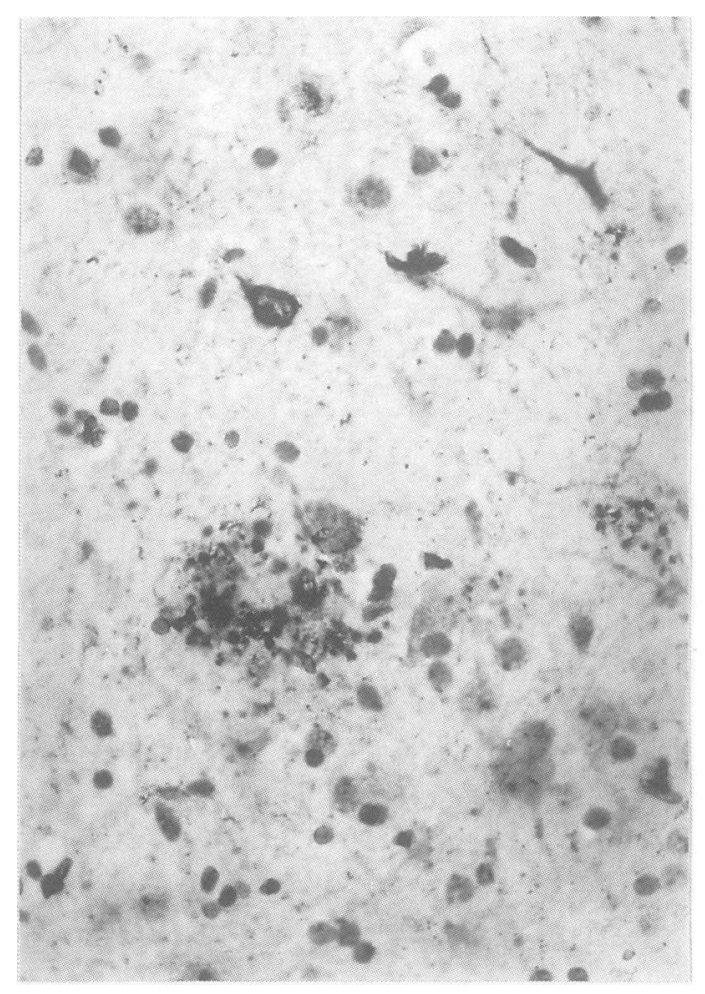

Figure SMI 34 MAb staining showing moderate immunostaining of neuritic plaques and neurofibrillary tangles (magnification $\times 400)$. 
reading comprehension, right-left discrimination, and finger localisation as well as acalculia and agraphia. She showed all the features of Balint's syndrome, including simultanagnosia, ocular motor apraxia, and impaired visuallyguided hand movements. Colour recognition and discrimination of unfamiliar faces were preserved, but she had impaired visual recognition of objects ( $20 \%$ correct) and familiar faces ( $30 \%$ correct). She also did poorly (more than 2 SDs below the standardised scores) in other complex visual perceptual tests (Hooper visual organisation test, visual form discrimination, and judgement of line orientation), and on constructional tasks (three dimensional block construction).$^{89}$ She also showed ideational apraxia, dressing apraxia, spatial disorientation, and loss of mental imagery for colours, objects, animals, and spatial associations. An electroencephalogram showed bilateral slow wave activity over posterior brain regions. Magnetic resonance imaging (MRI) revealed cortical atrophy restricted to the posterior parieto-occipital areas, with no evidence of atrophic changes in fronto-temporal regions (figure).

\section{Neuropathology}

After informed consent and to help in the diagnosis of the underlying neurological disorder a brain biopsy specimen was obtained from the right parietal cortex (Brodmann's area 7). Specimens were stained with haematoxylineosin, PAS, McMannus, Congo red, and Bielchowski silver impregnation. Immunocytochemistry was performed with a monoclonal antibody against paired helical filament (SMI $34 \mathrm{MAb}$ ). There was mild neuronal loss and a mild lipofucsin accumulation. Congo red and Bielchowski silver impregnations showed abundant neuritic plaques (NPs) (mean plaque count 8.6 per $250 \times$ field) and occasional neurofibrillary tangles (NFTs). There were also some immature NPs but no granuvacuolar degeneration. SMI $34 \mathrm{MAb}$ immunostained both NPs and NFTs (figure). There were no Pick bodies, Lewy bodies, subcortical gliosis, or status spongiosus.

\section{Discussion}

The syndrome of PCA does not necessarily imply Alzheimer's pathology as it may also be seen in cases of Pick's disease, progressive subcortical gliosis, and Creutzfeldt-Jakob disease (Heindenhain's type) ${ }^{2}$ Cognitive and perceptual deficits of similar characteristic to PCA, however, have been most often reported in the early and middle stages of $\mathrm{AD},{ }^{10-13}$ probably reflecting an initial, selective patho- logical involvement of the occipital and posterior parietal association areas. A recent neuropathological study of a subpopulation of $\mathrm{AD}$ patients presenting with a Balint's syndrome revealed prominent histopathological changes in parieto-occipital regions with little involvement of other neocortical areas (such as the prefrontal cortex) that are usually affected in typical cases of $\mathrm{AD} .^{14}$

Hof and co-workers recently suggested that Balint's syndrome in patients with $\mathrm{AD}$ results from a specific regional and laminar distribution of NPs and NFTs in the occipito-parietal association cortex with preferential degeneration of the dorsal occipito-parietal visual pathway. ${ }^{14}$ These structures were presumably affected in our patient. The coexistence of visual object agnosia, prosopagnosia, and letter-byletter reading, however, reflects additional degeneration of the ventral occipital-temporal visual pathway. Finally, the occurrence of spatial disorientation, dressing apraxia, ideational apraxia, anomic aphasia, and Gertsmann's syndrome suggests involvement of the adjoining inferior parietal lobes.

This work was supported in part by grants from the Instituto $D$ Tella (MLB), University of Buenos Aires, and a National Alliance for the Research in Schizophrenia and Depression Young Investigator Award (SES). We thank Dr G Herrera for allowing us to examine the patient; Dr L Lopez for neuropthalmological examination; and Dr C Bocaleri for performing the MRI.

1 Benson DF, Davis JR, Synder BD. Posterior cortical atrophy. Arch Neurol 1988;45:789-93.

2 Ross GW, Benson DF, Verity AM, Victoroff JI. Posterior cortical atrophy: neuropathological correlations. Neurology 1990;(suppl 1)40:200.

3 Blessed G, Tolimson BE, Roth $M$. The association between quantitative measures of dementia and of senile change in the cerebral gray matter of elderly subjects. Br J Psychiatry 1968;114:566-72.

4 Folstein MF, Folstein SE, McHugh PR. "Mini-Mental State": a practical method for grading the cognitive state of patients for the clinician.J Psychiat Res 1975;12: $189-98$.

5 Wechsler D. Wechsler adult intelligence scale. New York: Psychological Cooporation, 1981.

6 Wechsler D. A standardised memory scale for clinical use. $J$ Psychol 1945;19:87-95.

7 Kertesz A. The Western aphasia battery. New York: Grune and Stratton, 1982.

8 Hooper HE. The Hooper visual organisation test. Los Angeles: Western Psychological Services, 1958.

9 Benton AL, Hamsher KS, Varney NR, Spreen O. Contributions to neuropsychological assessment. A clinical manual. New York: Oxford Univ Press, 1983.

10 Schlotterer G, Moscovitch M, Crapper-McLachlan D. Visual processing deficits as assessed by spatial frequency contrast sensitivity and backward masking in normal ageing and Alzheimer's disease. Brain 1983;107:309-25.

1 Cogan DG. Visual disturbances with focal progessive dementing disease. Am J Ophthalmol 1985;100:68-72.

12 Nissen MJ, Corkin S, Buonanno FS, Growdon JH, Wray SH, Bauer J. Spatial vision in Alzheimer's disease: general SH, Bauer J. Spatial vision in Alzheimer's disease: general

findings and a case report. Arch Neurol 1985;42:667-71.
13 Mendez MF, Mendez MA, Martin R, Smyth KA, Whiterhouse PJ. Complex visual disturbances in Alzheimer's disease. Neurology 1990;40:439-43.

14 Hof PR, Bouras C, Constantinidis J, Morrison JH. Balint's syndrome in Alzheimer's disease: specific disruption of the occipito-parietal visual pathway. Brain Res 1989;493: 368-75. 